\title{
MACRO VERSUS MICRO THREAD DESIGN IMPLANT AND THEIR EFFECT ON THE STABILITY
}

\author{
Ahmed F. Ali Saleh ${ }^{1 *} B D S$, Ahmed S. Mohamed El- Mahallawy ${ }^{2} P h D$, Hala R. Ragab ${ }^{3} P h D$.
}

\begin{abstract}
INTRODUCTION: Dental implants are considered stable tools for replacing missing teeth, achieving long-term success rates above 90 percent, and their use in dental practice has become normal. One of the reasons to this success is the primary reliability of an implant. Implant design is one of the main factors that play a role in stability.

OBJECTIVES: The purpose of this study was to compare the role of the Macro thread design and the Micro thread design on implant stability through the use of the Resonance Frequency Analysis (RFA).

MATERIALS AND METHODS: The study was carried out on 6 patients, each of these patients has missing teeth on each side of posterior area in the lower jaw. All the right sides received Micro thread design implant (dentium implant,) and the left side received Macro thread design implant (Megagen AnyRidge). After dental implants were placed in their sites, the stability was measured by using the Resonance Frequency Analysis (RFA) to assess the stability of the two types of implants at three times periods: At the time of placing the implant, 3 months and 6 months.

RESULTS: The mean implant Stability value for Group A was 70.57 \pm 5.74 immediately post-operatively, on the 3 month to $77.14 \pm 6.74$ and reach $84.29 \pm 6.02$ on the 6 month, for Group B was $63.29 \pm 6.58$ immediately post-operatively, on the 3 month $70.57 \pm 4.69$ and on the 6 month $77.14 \pm 4.53$. The mean bone density values for Group A at 3 months was $481.98 \pm 51.78$ and at 6th month was $504.28 \pm 47.50$, in Group B the 3 months was $439.54 \pm 70.49$ and at 6th month was $463.83 \pm 74.44$.
\end{abstract}

CONCLUSION: The Micro thread design implants shows higher stability than Macro thread design.

KEYWORDS: Osseo integration, Implant design, Dentium implant, Megagen AnyRidge, Resonance Frequency designs available Analysis, CBCT. RUNNING TITLE: Macro, micro thread design, implant stability.

1 Resident at Conservative Oral and Maxillofacial Surgery Department, Faculty of Dentistry, Alexandria University, Alexandria, Egypt.

2 Professor of Oral and Maxillofacial Surgery, Faculty of Dentistry, Alexandria University, Egypt.

3 Assistant Professor of Oral and Maxillofacial Surgery, Faculty of Dentistry, Alexandria University, Egypt.

*Corresponding author

E-mail: ahmedfouadali148@gmail.com

\section{INTRODUCTION}

Dental implants are considered one of the most well-known effective ways of missing teeth replacement. Realizing success rates more than $90 \%$ in the long follow up period, and their use has become familiar in dental practice. The first step of successful osseointegration is the primary stability of an implant (1).

In the 1960s the modern dental implant has become one of the critical choices of the treatment option in the replacement of missing natural teeth. Since then, the dental implant industry has recently seen enormous development in a vast number of manufacturers and several designs are available.

Recently, a variety of implant lengths, surfaces, body designs, platform connections, thread forms, and body designs are available, where these variations in implant designs available can aid in primary stability (initial stability) and Osseointegration. It has been reported that implant design is a vital parameter for obtaining primary stability (2). Also, the presence of Osseointegration is crucial to evaluate implant success (3).

Various mechanical factors form the effective connection between an implant and its surrounding bone. One of these factors is the design of implants which Specifies stability and distribution of pressure during the process of osseointegration (3), where the implant designs contain macroscopic features include (body design and thread geometry) and the 
This study proposes that this Macro thread design may have similar results to Micro thread design in their effect on the stability of the implant.

\section{MATERIALS AND METHODS}

Appropriate ethical clearance was obtained from the institution at which the study was conducted. Where all patients received thorough explanations about the planned treatment and its potential risks and complications and signed a written informed consent form before being enrolled in the study. It was also mentioned that the patient had the right to withdrawal from the study anytime without any consequences. Ethical approval for this study was obtained from the research ethics committee, Faculty of Dentistry, Alexandria University before beginning the study.

\section{Study design}

The present study was a randomised controlled clinical trial (split mouth design) performed in six patients with 14 dental implants. Each patient in attendance received one implant on each side, except for one of those patients who received two implants on each side, (Seven of the implants were Macro thread design Megagen Any Ridge and the other seven were Micro thread Dentium implants). The age range of the participating patients was between $30-50$ years of age. All cases of missing mandibular teeth indicated for implant placement by two-stage surgery. Patients have been chosen from the Outpatient Clinic of the Department of Oral and Maxillofacial Surgery, Faculty of Dentistry, University of Alexandria. The dental implants were divided into two groups (A and B): Group (A): On the right side consist of 7 implants with Micro thread design.

Group (B): On the left side consist of 7 implants with Macro thread design.

\section{Criteria for patient's selection}

Inclusion criteria: A healthy condition that initiates bone healing, a mandibular edentulous site for implant placement and a sufficient volume of bone at the implant placement site.

Exclusion Criteria: Systemic illness, drug or habit considered to have a negative effect on bone healing and/or dental implant success: (poorly controlled diabetes [ HbA1c > $8 \%$ ], heavy smoker, para dental habits, history of use of bisphosphonate, history of radiation therapy of the head and neck affecting the proposed implant Site, current use of medication adverse to healing [e.g. corticosteroids, chemotherapy].

\section{Materials}

Group (A): Dentium Implant System (Super Line)

- Micro thread design was placed on the right side.

- Tapered design, Extended cutting edge, Double thread design.

- The size of the implant that was placed in this study 4.0 mm diameter and 10mm length.

Group (B): Megan Implant System (AnyRidge)

- Macro thread design was placed on the left side.

- Incorporates a novel thread design, which includes rounded, non-cutting edge, wide thread depth, and increased thread pitch compared to a conventional thread design.

- The size of the implant that was placed in this study 4.0 mm diameter and $10 \mathrm{~mm}$ length.

\section{Surgical kit}

This includes the surgical instruments used for flap reflection, an electric motor with irrigation system, drills with different sizes for each system, guide drill, drill extension countersink, depth gauge, and parallel pins, drivers, hand wrench, ratchet wrench, a contra-angle handpiece, and Osstell.

\section{Pre- operative Stage}

Each patient was evaluated by taking appropriate history and thorough clinical examination. Initial periodontal treatment was completed, including scaling and oral hygiene training.

\section{Preliminary assessment}

For both arches and diagnostic test designs, the primary alginate impression was taken, clinically as well as on the study design, the inter-arch relationship, interocclusal space that could accommodate the implant abutment, and potential crown restoration were evaluated.

Vacuum-Formed surgical guidance stent was manufactured and in the stent opposite the missing tooth a hole was drilled indicating the implant's location.

Radio-graphical examination

- For the examination of bone height, panoramic radiography was obtained to detect the presence of any pathology at the implant site.

- Cone-beam computed tomography (CBCT) was done to detect: (buccolingual width of the ridge, length, and diameter of the implant, bone density, approximation to vital anatomical structures. (Figure 1)

Surgical Procedures

The two groups were operated under local anesthesia (mandibular nerve block technique), using Articaine with vasoconstrictors (1:200 000).

- Mid crestal incision was done down to the bone with a blade No.15 was utilized, including one tooth on either side of the proposed implant site. (Figure 2A, 3A)

- Sharp dissection was performed by a periosteal elevator.

- Implant placement procedures are done in compliance with the manufacturer's instructions.

- The electric motor was used to prepare the implant site together with a low-speed high torque handpiece externally irrigated.

Sterile saline was used during the preparation of the implant site for external irrigation.

- The preparation of the bone site was undertaken using the $1 \mathrm{~mm}$ pilot drill with external irrigation. It adjusted the electric motor to $1000 \mathrm{rpm}$.

- The initial drill $2 \mathrm{~mm}$ was used to reach to the predetermined length.

- Sequential drilling has been done up to the final drill. (Figure 2B, 3B)

- The depth gauge and path indicator were used to assess the osteotomy site depth and to test the implant's parallelism. (Figure 2C, 3C)

- The implant was inserted manually into its bed under moderate apical pressure until it stopped. Then the implant was inserted into the final mounting depth by a ratchet wrench. (Figure 2D, 3D)

- Osstell smart peg connected to the fixture and initial stability measurement taken. (Figure 2E, 3E)

- Cover screw was inserted and secured into the implant's occlusal opening. (Figure 2F, 3F) 
- Flap repositioned after the surgical site was irrigated and dry.

- 3.0 black silk suture material used to suture the flap.
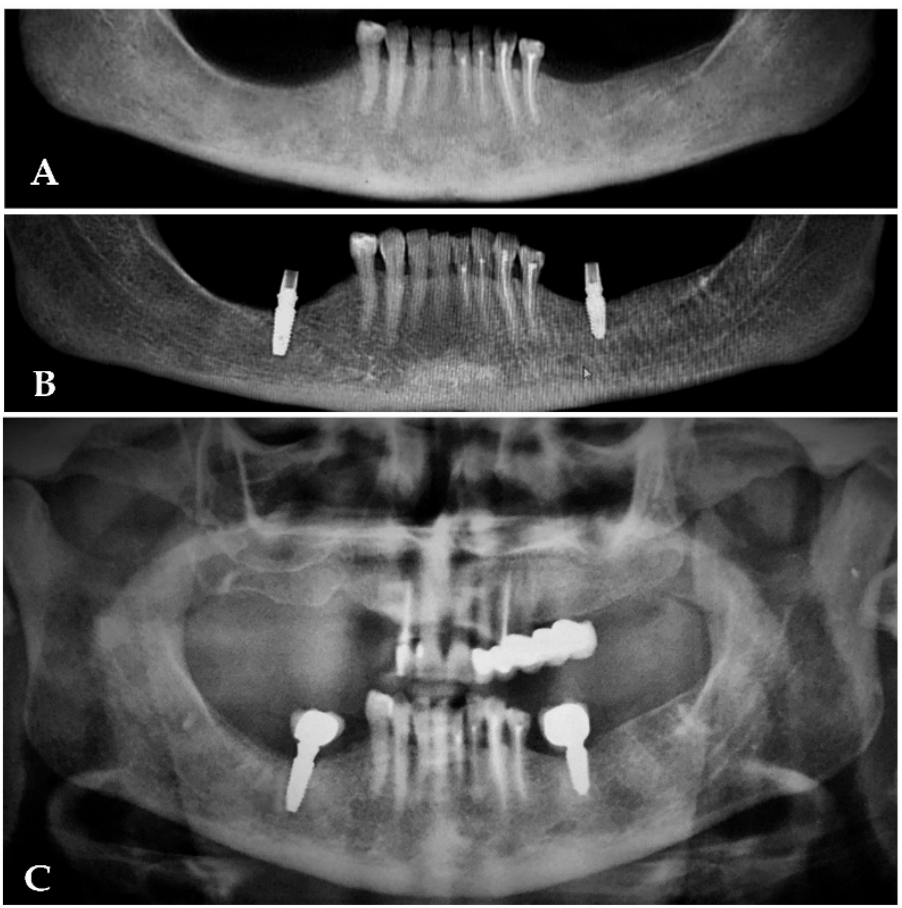

Figure (1): (a) Pre-operative CBCT- (b) Radiograph after 3 months postoperative CBCT- (c) Radiograph with final restoration after 6 months postoperative.
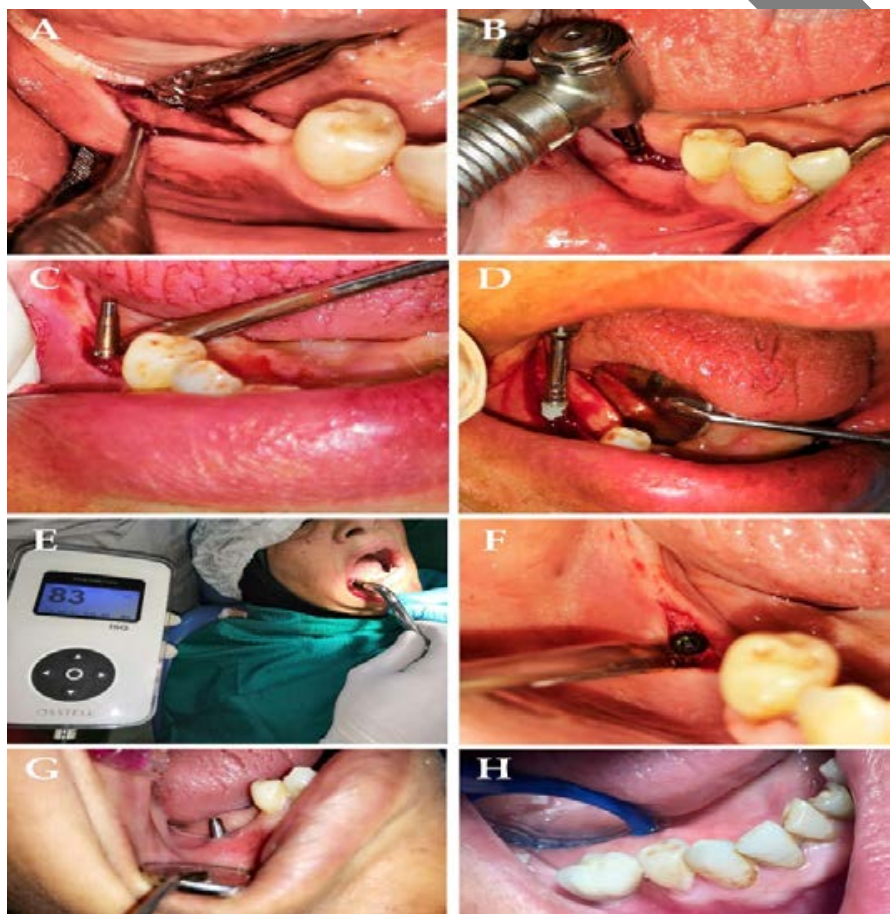

Figure (2): (a) Mid Crestal Flap Reflection. (b) Drilling the bone. (c) Parallel Pin. (d)Implant placement (Dentium dental implant) Group A. (e) Osstell measuring. (f)Implant with cover screw. (g)Abutment in place right side (Dentium dental implant). (h) Final porcelain restoration lower right six.
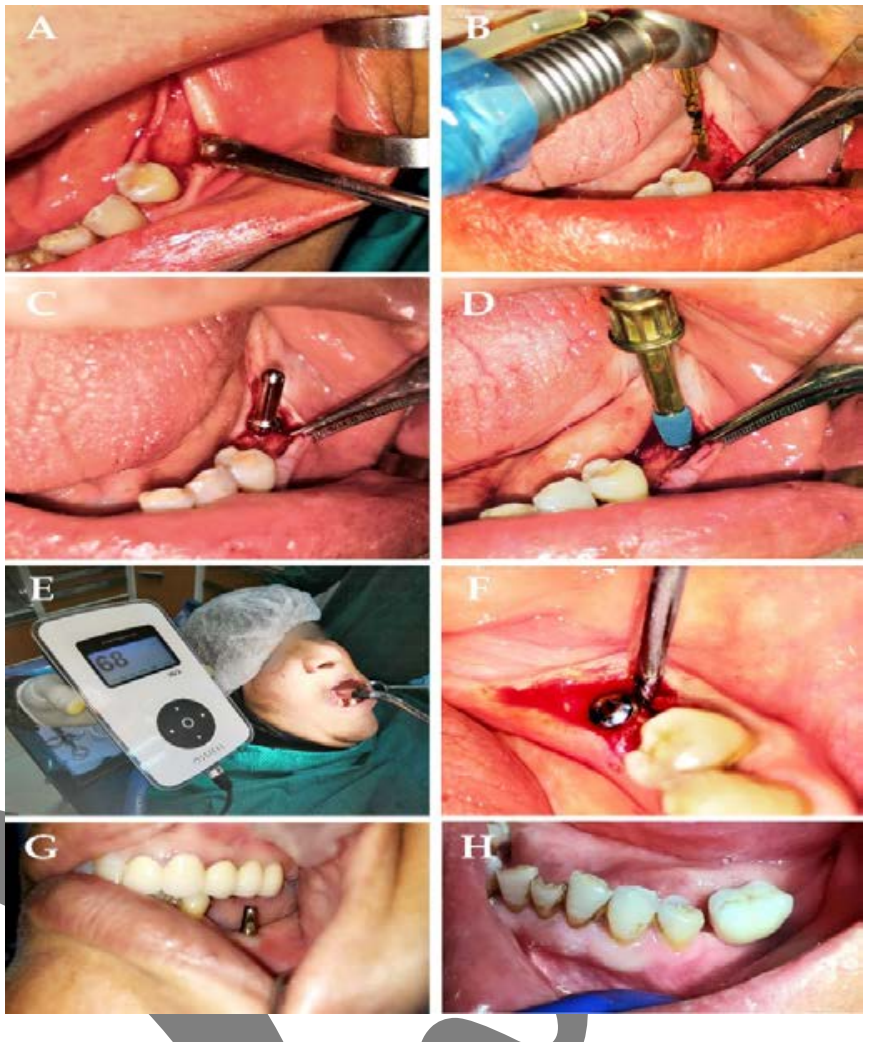

Figure (3): (a) Mid Crestal Flap Reflection. (b) Drilling the bone. (c) Parallel Pin. (d)Implant placement (Maga-Gan dental implant) Group A. (e) Osstell measuring. (f)Implant with cover screw. (g) Abutment in place right side (Maga-Gan dental implant). (h) Final porcelain restoration lower left six.

Post-surgical phase

The patient was instructed to rinse their mouth with antiseptic chlorhexidine mouthwash 3 times a day starting from the second day postoperatively and continued for 2 successive weeks.

Postoperative panoramic x-rays were taken to ensure proper placement of the implant and the implant's relationship to opposing landmarks or surrounding structures.

\section{Follow up phase}

The following clinical parameters were recorded immediately after loading (baseline) and at the end of the study:

\section{Presence or absence of pain or sensitivity}

The patient comfort was assessed using the Visual Analogue Scale (VIS) (6) of 0 to 10.

\section{Probing depth (PD)}

According to Harvard Conference (7), the probing pocket depth around the implant was measured on the four dimensions of the implant's facial, palatal and proximal surfaces using a mm periodontal probe graduation.

\section{Healing index score}

HI recording was done on the post-surgery first-week. The HI rates healing based on redness, tissue appearance, swelling, suppuration, and epithelialization.

Landry, Turnbull, and Howley (8) have developed a healing index to explain the degree of medical healing following periodontal surgery, and it has also been recently updated to be used for socket healing. For post-extraction score (0/1) with a total score of seven, with a total score of 7: presence/absence of redness; presence/absence of granulation tissue; presence/absence 
of suppuration; presence/absence of swelling; degree of tissue epithelialization (partial/complete); presence/absence bleeding; presence/absence of pain on palpation.

\section{Stability Evaluation}

When evaluating implant stability and osseointegration, The Osstell was measuring stability at three and six months.

The result was provided as a 1-100 Implant Stability Quotient (ISQ) value $(9,10)$. Osstell is a non-invasive procedure first used in 1996. This consists of a small L-shaped transducer fastened to the implant or the transmucosal abutment using a screw. There is a vertical beam connected to this transducer with two piezoceramic components. One of the piezoceramic elements produces a vibration consisting of a small, 5 to 15 $\mathrm{kHz}$ sinusoidal signal in $25 \mathrm{~Hz}$ steps. The other piezoceramic component analyzes the transducer's response to the vibration. The higher the ISQ the more stable is the implant.

\section{Radiographic Evaluation}

Cone Beam Computed Tomography (CBCT): is a threedimensional imaging technology that offers less radiation dosage reportedly up to 15 times lower than those of conventional CT scans.

Assessment of bone density after three and six months from dental implants placement using CBCT, after dental implant placement bone density was measured around the dental implant site by using Hounsfield unit $\mathrm{HU}$ to detect the osseointegration.

Three controlled and standardized dimension square areas were selected just mesial, distal and apical to the implant including the Bone-implant interface, Mean, Standard deviation, Minimum and Maximum readings were automatically displayed by the system "On-demand 3D" and used for statistical analysis.

\section{Prosthetic phase}

The cover screw was removed and the healing abutment was tightened at the third

month. (Figure 2G, 3G)

After 4 months of this study the patients were recalled for delivery of the definitive restoration, in which impressions were taken and sent to the laboratory for the fabrication of the final restoration. (Figure $2 \mathrm{H}, 3 \mathrm{H}$ )

Statistical analysis

Statistical analysis Data were fed to the computer and analyzed using version 20.0 (11) of the IBM SPSS software package (Armonk, NY: IBM Corp). Using range, mean, standard deviation and median, quantitative data were described. By the Kolmogorov-Smirnov method, the distribution of quantitative variables was checked for normality. The paired t-test was used to compare two periods of quantitative variables normally distributed, while the ANOVA of repeated measurements was used to compare more than two phases or stages with the Bonferroni Post Hoc test. The Friedman test has been used with Dunn's Post Hoc Test of abnormally distributed quantitative variables. The results obtained were calculated at the rate of $5 \%$.

\section{RESULTS}

The present split-mouth study was conducted on 6 patients with 14 implants, each case received two implants with specific thread design in the lower jaw (on the left side Macro thread - on the right side Micro thread model), all cases selected from the oral and maxillofacial surgery department's outpatient clinic, Faculty of Dentistry, Alexandria University.

Alexandria Dental Journal. Volume 46 Issue 1 Section A
Their ages ranged from 30 to 50 years, the teeth that this study replaced were multi-rooted mandibular teeth, all patients were free of any local or systemic infection.

The 14 implants were divided into two groups, 7 implant Macro thread design: (Mega Gen) and 7 implants Micro thread design (Dentium)

Group (A): Included seven Micro thread implants that were placed on the right side of the lower jaw.

Group (B): Included seven Macro thread implants that were placed on the left side of the lower jaw.

\section{Clinical evaluation}

\section{Presence of pain}

Using visual analog scale (VAS) from 0 to 10 (“0” is pain-free and " 10 " is unsustainable pain), the pain was evaluated daily for first week. After surgery, patients reported mild pain at the surgical site, which had gradually subsided after the procedure by the fourth day.

\section{Probing depth}

Probing depth was measured for all implant axial surfaces, statistical analysis of sampling depth scores for all patients was performed. Collecting and tabulating data. The mean Probing depth scores of group A on the third month were $1.82 \pm 0.47$ with a minimum documented value of 1.0 and a maximum recorded value of 2.5, while the mean Probing depth scores of group B were $2.00 \pm 0.65$ with a minimum recorded value of 1.50 and a maximum recorded value of 3.0 . This difference in the probing depth score $(\mathrm{P} 2=<0.001)$ was found to be statistically significant. The mean probing depth scores of Group A on the sixth month were $1.36 \pm 0.38$ with a minimum reported value of 1.0 and a maximum recorded value of 2.0, while the mean probing depth scores of the control group were $1.64 \pm 0.56$ with a minimum recorded value of 1.0 and a maximum recorded yalue of 2.5. This difference in the probing depth score (P2= around 0.001) was found to be statistically significant. (Figure 4 A, B) (Table 1)

Table (1): Comparison between the studied groups according to peri-implant probing depth throughout the study period.

\begin{tabular}{|l|c|c|c|c||}
\hline \multirow{2}{*}{$\begin{array}{c}\text { Peri-implant } \\
\text { probing } \\
\text { depth }\end{array}$} & \multicolumn{2}{|c|}{ Group A (n = 7) } & \multicolumn{2}{c|}{ Group B (n = 7) } \\
\cline { 2 - 5 } & $\begin{array}{c}\mathbf{3}^{\text {rd }} \\
\text { month }\end{array}$ & $\begin{array}{c}\mathbf{6}^{\text {th }} \\
\text { month }\end{array}$ & $\begin{array}{c}3^{\text {rd }} \\
\text { month }\end{array}$ & $\mathbf{6}^{\text {th }}$ month \\
\hline Min. - Max. & $1.0-2.50$ & $1.0-2.0$ & $1.50-3.0$ & $1.0-2.50$ \\
Mean \pm SD. & $1.82 \pm 0.47$ & $1.36 \pm 0.38$ & $2.00 \pm 0.65$ & $1.64 \pm 0.56$ \\
Median & 2.00 & 1.50 & 1.50 & 1.50 \\
\hline
\end{tabular}

\section{Healing index score}

Healing index score was evaluated from the first week till a month, none of the implants showed any (redness, granulation tissue, suppuration, swelling and bleeding) score was (0).

\section{Implant Stability Evaluation}

At the time of implant insertion, the implant stability measurement was examined 3 times, 3rd month and 6th month for both groups using the Resonance Frequency Analysis through the Osstell (ISQ) system.

Group (A):

The implant stability for patients of (Group A) who received Micro thread implant placement and the results were measured by using the Resonance Frequency Analysis. 
From the table above its shown that the maximum stability measured at the time of operation was of (ISQ) $=80$, (ISQ) units and the minimum measured was 65 (ISQ) units with an average mean and standard deviation $=70.57 \pm 5.74$.

Then after 3 months shows that the maximum stability measured was of (ISQ) $=84$ (ISQ) units and the minimum measured was 68 (ISQ) units with an average mean and standard deviation $=77.14 \pm 6.74$.

After 6 months that the maximum stability measured was of $($ ISQ) $=91$ (ISQ) units and the minimum measured was 74 (ISQ) units with an average mean and standard deviation = $84.29 \pm 6.02$

Average values for all cases of group A, Min. - Max $=69-85$ ISQ units, mean and standard deviation for all cases of group A were $77.33 \pm 5.93$ ISQ units, where the average calculated mean value for those cases was 78.3 ISQ units.

\section{Group (B):}

The implant stability for patients of (Group B) who received Macro thread implant placement and the results were measured by using the Resonance Frequency Analysis.

From the table above its shown that the maximum stability measured at the time of operation was of (ISQ) $=74$, ISQ units and the minimum measured was 56 ISQ units with an average mean and standard deviation $=63.29 \pm 6.58$

Then after 3 months shows that the maximum stability measured was of ISQ $=77$ ISQ units and the minimum measured was 65 ISQ units with an average mean and standard deviation $=70.57 \pm 4.69$.

After 6 months the maximum stability measured was 83 ISQ units and the minimum measured was 71 ISQ units with an average mean and standard deviation $=77.14 \pm 4.53$.

The average value of all group B cases, Min. - Max $=65-77$ ISQ units, mean and standard deviation was 70.33 \pm 4.29, Where the average calculated mean value for those cases was 69.33 ISQ units.

Comparing the above stability results for the two groups studied, it was shown that the average mean and standard deviations of the stability for all Group A cases receiving Micro thread design implants were 77.33 \pm 5.93 ISQ units. That is greater than the average mean and standard deviation of the stability for all Group B cases receiving Macro thread implants, which was 70.33 \pm 4.29 ISQ units. (Figure 5) (Table 2)

\section{Radiographic Evaluation}

\section{Bone density}

The bone density was measured using the On demand program. The mean was calculated after 3, 6 months; show that there was a significant increase in the mean bone density by time at the two periods of follow up in both groups for all cases.

\section{In group $A$}

The maximum bone density measured after 3 months was 532.8 and the minimum 388.43 measured with an average mean and standard deviation $=481.98 \pm 51.78$.

After 6 months that the maximum bone density measured was 582.8 and the minimum measured was 442.47 with an average mean and standard deviation $=504.28 \pm 47,50$.

\section{In group B}

The maximum bone density measured after 3 months was 561.53 and the minimum 384.03 measured with an average mean and standard deviation $=439.54 \pm 70.4$.
After 6 months that the maximum bone density measured was 572.83 and the minimum measured was 370.57 with an average mean and standard deviation $=463.83 \pm 74.44$.

The average value of all cases of group $A$, mean bone density value was 451.69, with SD 70.09. Cases of group B mean bone density value was increased to 493.13, with SD 47.35.

Table (2): Comparison between the two studied groups according to stability.

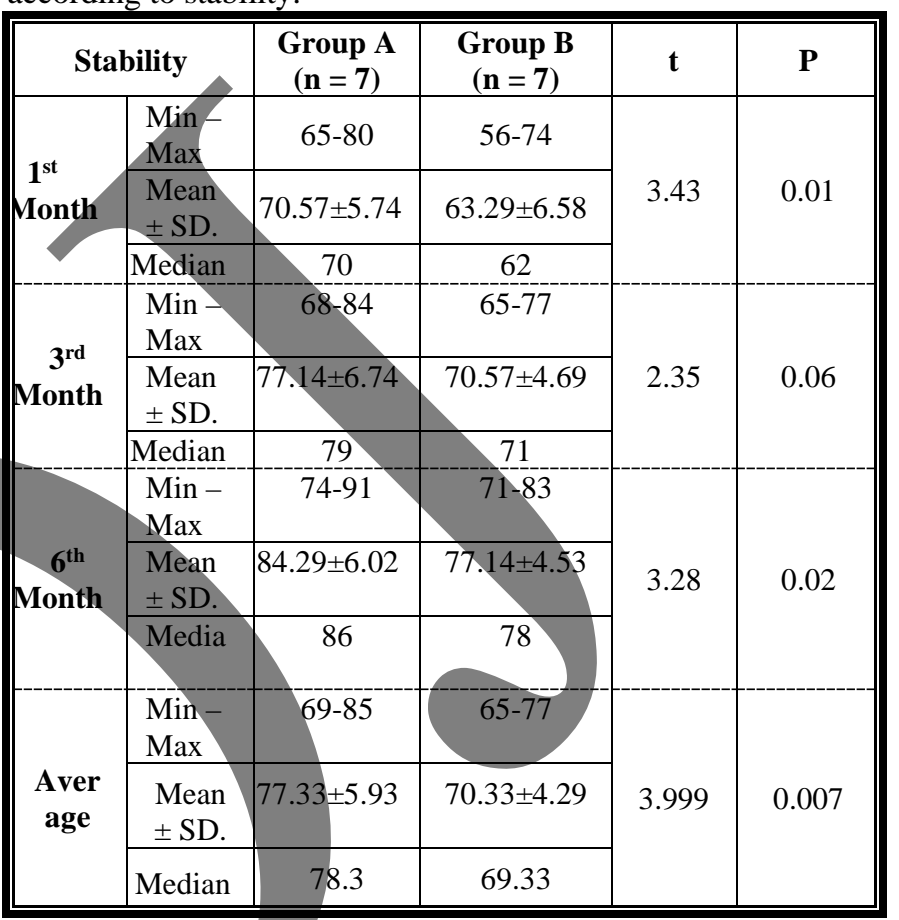

$\mathbf{t}=\mathrm{T}$ test to determine if there is a significant difference between the means of two groups.

$\mathbf{p}=\mathrm{P}$ value this value, which determines the "significance of results" in hypothesis testing, tells us the how strong a claim or null hypothesis is. Significance level used is 0.05.

Comparing the above-mentioned bone density findings for the two groups analyzed, it was shown that the average of mean and standard deviations in bone density for Group A cases receiving Micro thread pattern implants were $493.13 \pm 47.35$. This is greater than the average mean and standard deviation of bone density for all Group B cases with macro thread implants, which was $451.69 \pm 70.09$. (Figure 6) (Table 3)

Table (3): Mean bone density values for different periods for group (A\&B)

\begin{tabular}{||c|c|c|c|c||}
\hline \multirow{2}{*}{ Bone density } & \multicolumn{2}{|c|}{ Group A (n = 7) } & \multicolumn{2}{c|}{ Group B (n = 7) } \\
\cline { 2 - 5 } & $3^{\text {rd }}$ & $\mathbf{6}^{\text {th }}$ & $3^{\text {rd }}$ & $\mathbf{6}^{\text {th }}$ month \\
& month & month & month & \\
\hline Min. - & $388.43-$ & $442.47-$ & $384.0-$ & $370.57-$ \\
Max. & 532.8 & 582.8 & 561.5 & 572.8 \\
Mean \pm & 481.98 & 504.28 & 439.54 & $463.83 \pm$ \\
SD. & \pm 51.78 & \pm 47.50 & \pm 70.49 & 74.44 \\
Median & 486.30 & 507.90 & 399.80 & 444.17 \\
\hline
\end{tabular}




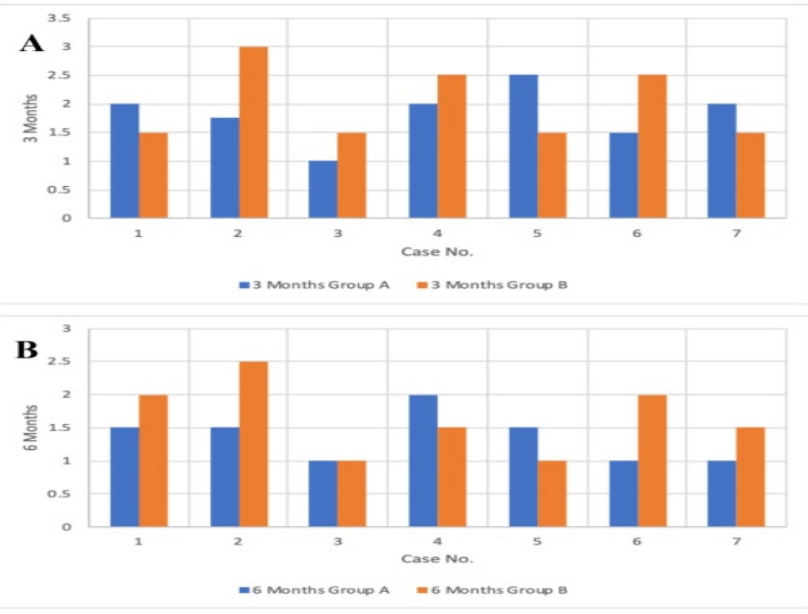

Figure (4): (a) Evaluation of probing depth for the 3 Months period for Group (A) and (B), (b)Evaluation of probing depth for the 6 Months period for Group (A) and (B).

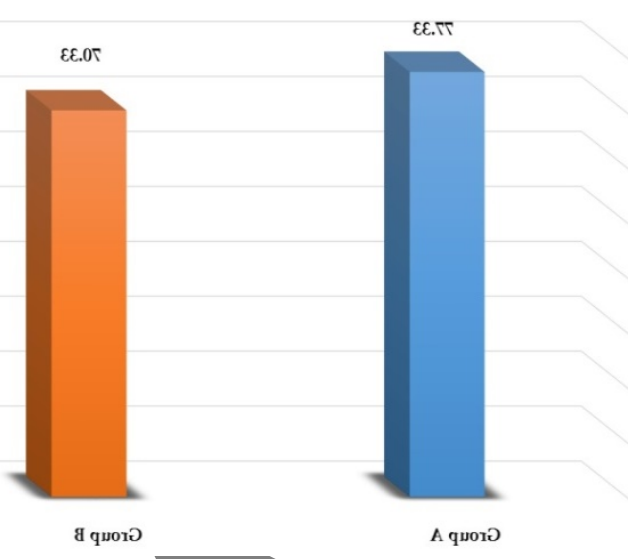

08

\section{or}

0

02

ot

$0 \varepsilon$

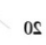

or

Figure (5): Comparison between mean stability values for different periods for groups $(A \& B)$.

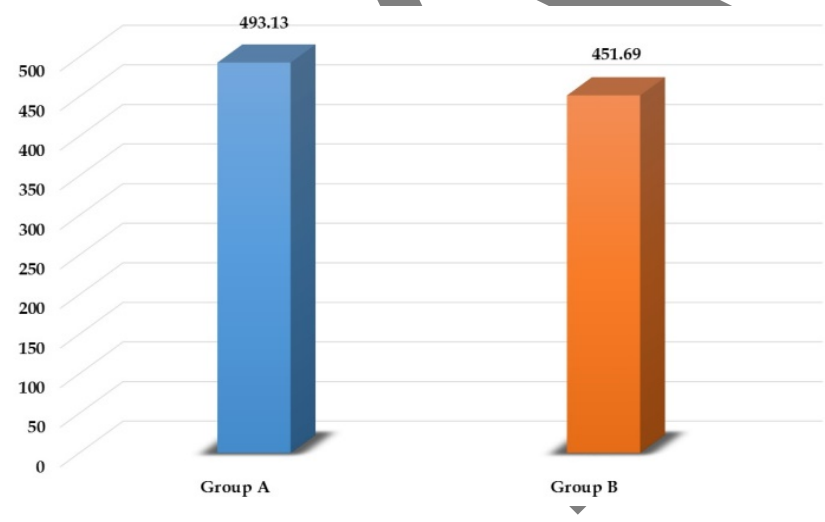

Figure (6): Comparing between the bone density values for different periods for group (A\&B).

\section{DISCUSSION}

Stability is one of the key factors in the successful treatment of dental implants. Branemark's osseointegration, meaning the structural and functional coordination between the implant and the underlying vital bone, is focused on long-term endosseous dental implant.
Bone density and implant stability are important factors for implant Osseointegration which have been widely demonstrated by several authors. It is suspected that the lack of primary stability is the cause of early implant failure (12), Stability depends on bone strength, surgical procedure and the micro- and macro-design of the implant used; thus, implant stability is the key to clinical success (13-15).

The present study aimed to compare two different implant profiles Micro thread "Narrow pitch" implants and Micro thread "wide pitch" implant was tested in the posterior area of the lower jaw, where we use this study to evaluate the influence of implant thread design on stability and osseointegration process. The purpose of this hypothesis is that selecting implant design features that increase surface area for contact that may improve the stability and boneimplant contact.

In this study, six patients with missing mandibular posterior teeth were chosen from the Outpatient Clinic, Faculty of Dentistry, Alexandria University, Department of Oral and Maxillofacial Surgery. The chosen patients were free from any untreated systemic disorders or conditions that might hinder the surgical or healing process of the implant.

Bornstein, Cionca et al., (16) investigating whether systemic diseases with / without systemic medicines increased the risk of implant failure and thus decreased the effectiveness and survival of dental implants, stated that the level of evidence indicating absolute and relative implant therapy contraindications due to systemic diseases was small.

Patients with bruxism, heavy smokers, chemotherapy or radiotherapy patients, and patients with immune suppression were the ones omitted from this study. Gómez-de Diego et al. (17) conducted this study to analyze clinically affected patients ' indications and contraindications of dental implants and concluded that the use of nicotine and head and neck radiotherapy is associated with increased loss of dental implants. CBCT was used to measure bone density due to its sensitivity, lower radiation exposure and lower cost compared to CT.

The mean bone density in our sample increased significantly towards the post-operative six-month period. The results show that there was a significant increase in the mean bone density by time at the two periods of follow up in both groups for all cases:

The average value of all cases of group A, mean bone density value was 493.13, with SD 47.35, the Average value of all cases of group $\mathrm{B}$, mean bone density value was increased to 451.69, with SD 70.09 .

However, in 2011, Naser et al. (18) compared continuous films taken over time at different time scales to research alveolar bone changes and bone density around dental implants, finding that the average density obtained at different standard densitometry stages showed a gradual increase in bone density throughout the phase.

Implant stability was tested through the Osstell ISQ method using Resonance Frequency Analysis (RFA), RFA was chosen as a non-invasive and reliable approach to assess variation over time in implant stability. RFA registrations are directly related to the stability of the implant in the surrounding bone: new bone apposition at the implant-bone interface may be expressed during healing and increase of the ISQ values (19-20). 
The normal range of ISQ values commonly recorded for primary stability implants is between 60 and 80, although a consensus has not been developed on the ISQ threshold below which an implant should not be considered stable.

Meredith et al. (21) concluded that RFA is a method that can serve as a valuable research technique and is useful in studying the operation of implants in the surrounding tissue.

Jaramillo et al. (22) have stated that in Osstell Mentor and Osstell ISQ, Resonance frequency analysis systems show almost perfect reproducibility, repeatability, and accuracy.

Results from this study showed that "Narrow pitch" Micro thread implants obtained higher primary stability values compared to "wide pitch" Macro thread implants measured with RFA.

Our result shown the average value of stability measured for all group A cases, Min.-Max = 69-85 (ISQ) units, the overall mean and standard deviation are $77.33 \pm 5.93$ (ISQ) units for all group A cases and the average value of all cases of group B, Min. - Max =65-77 (ISQ) units, the average mean and standard were $70.33 \pm 4.29$.

These results were in accordance with similar studies which discussed similar relation and found that smaller pitch implant design increased bone-implant contact and primary stability. Also, similar results were obtained by other studies. Implants with smaller pitch are beneficial by increasing BIC (23).

In 2012, Lan et al., (24) found that the loading form is the main factor affecting the pressure distribution and that the thread pitch above $0.8 \mathrm{~mm}$ is more appropriate for screwed implants in biomechanical consideration.

Kong et al., (25) thread pitch plays a greater role than buccolingual load in protecting dental implants under axial load. The optimum configuration in a cylinder implant should be the thread pitch exceeding $0.8 \mathrm{~mm}$, but excessive pitch should also be avoided

Abuhussein et al., (26) conclude that attaching threads or micro threads to an implant's crystal module may potentially lead to the bone's interaction with implants as well as to the conservation of marginal bone.

The Macro thread implants show lower primary stability comparing with the Micro thread implants, which does not match the results of other authors, Gehrke et al,, (27) discussed a similar relation, and he found that greater primary stability with wide pitch compared to narrow pitch, Elitsa et al., (28) found that better primary stability with a higher thread profile, In the early post-operative healing phase, McCullough and Klokkevold (29) noticed macro-thread structure appear to play a role during implant stability as assessed by RFA.

\section{CONCLUSION}

According to this study, the geometry of dental implant threads affects the stability and BIC of the Micro thread design implants shows higher stability (as calculated using ISQ) than the Macro thread design. On the other hand, the bone density of the Micro thread design appears slightly higher than that of the Macro thread design.

\section{CONFLICT OF INTERSET}

The authors declare that they do not have any conflicts of interest.

\section{REFERENCES}

1. Gehrke S, da Silva U, Del Fabbro M. Does Implant Design Affect Implant Primary Stability? A Resonance Frequency Analysis-Based Randomized Split-Mouth Clinical Trial. J Oral Implantol. 2015;41:e281-e6.

2. Javed F, Ahmed H, Crespi R, Romanos G. Role of primary stability for successful osseointegration of dental implants: Factors of influence and evaluation. Interv Med Appl Sci. 2013;5:162-7.

3. Ryu H, Namgung C, Lee J, Lim Y. The influence of thread geometry on implant osseointegration under immediate loading: a literature review. J Adv Prosthodont. 2014;6:547-54.

4. McCullough J, Klokkevold P. The effect of implant macro-thread design on implant stability in the early postoperative period: a randomized, controlled pilot study. Clin Oral Implants Res. 2017;28:1218-26.

5. Chowdhary R, Halldin A, Jimbo R, Wennerberg A. Influence of Micro Threads Alteration on Osseointegration and Primary Stability of Implants: An FEA and In Vivo Analysis in Rabbits. Clin Implant Dent Relat Res. 2015;17:562-9.

6. Rathmell JP, Hill B. Wall and Melzack's Textbook of Pain, 5th E-dition. Anesth Analg. 2006;102:1914.

7. Schnitman P, Shulman L. Recommendations of the consensus development conference on dental implants. J Am Dent Assoc. 1979;98:373-7.

8. Landry RG, Turnbull RS, Howley T. Effectiveness of benzydamyne $\mathrm{HCl}$ in the treatment of periodontal postsurgical patients. Res Clin Forums. 1988;10:105-18.

9. Sennerby L, Meredith N. Implant stability measurements using resonance frequency analysis: biological and biomechanical aspects and clinical implications. Periodontol 2000. 2008; 47:51-66.

10. Meredith N, Alleyne D, Cawley P. Quantitative determination of the stability of the implant-tissue interface using resonance frequency analysis. Clin Oral Implants Res. 1996;7:261-7.

11. Brisman DL. The effect of speed, pressure, and time on bone temperature during the drilling of implant sites. Int J Oral Maxillofac Implants. 1996;11:35-7.

12. Esposito M, Hirsch J, Lekholm U, Thomsen P. Biological factors contributing to failures of osseointegrated oral implants, (I). Success criteria and epidemiology. Eur J Oral Sci. 1998;106:527-51.

13. Gapski R, Wang H, Mascarenhas P, Lang N. Critical review of immediate implant loading. Clin Oral Implants Res. 2003;14:515-27.

14. Sakoh J, Wahlmann U, Stender E, Nat R, Al-Nawas B, Wagner W. Primary stability of a conical implant and a hybrid, cylindric screw-type implant in vitro. Int J Oral Maxillofac Implants. 2006;21:560-6.

15. Rozé J, Babu S, Saffarzadeh A, Gayet-Delacroix M, Hoornaert A, Layrolle P. Correlating implant stability to bone structure. Clin Oral Implants Res. 2009;20:1140-5.

16. Bornstein MM, Cionca N, Mombelli A. Systemic conditions and treatments as risks for implant therapy Int $\mathrm{J}$ Oral Maxillofac Implants. 2009;24:12-27.

17. Gomez-de Diego R, Mang-de la Rosa M, Romero-Perez M, Cutando-Soriano A, Lopez-Valverde-Centeno A. Indications and contraindications of dental implants in 
medically compromised patients: Update. Med Oral Patol Oral Cir Bucal. 2014;19:e483-9.

18. Naser AZ, Etemadi S, Rismanchian M, Sheikhi M, Tavakoli M. Comparison of Conventional and Standardized Bone Densitometry around Implants in Periapical Radiographs during a Three Months Period. Dent Res J (Isfahan). 2011;8:33-8.

19. Sim CP, Lang NP. Factors influencing resonance frequency analysis assessed by Osstell ${ }^{\mathrm{TM}}$ mentor during implant tissue integration: I. Instrument positioning, bone structure, implant length. Clin Oral Implants Res. 2010;21:598-604.

20. Kessler-Liechti G, Zix J, Mericske-Stern R. Stability measurements of 1-stage implants in the edentulous mandible by means of resonance frequency analysis. Int $\mathrm{J}$ Oral Maxillofac Implants. 2008;23:353-8.

21. Meredith N, Shagaldi F, Alleyne D, Sennerby L, Cawley P. The application of resonance frequency measurements to study the stability of titanium implants during healing in the rabbit tibia. Clin Oral Implants Res. 1997;8:234-43.

22. Jaramillo R, Santos R, Lázaro P, Romero M, Rios-Santos J, Bullón P, et al. Comparative analysis of 2 resonance frequency measurement devices: Osstell Mentor and Osstell ISQ. Implant Dent. 2014;23:351-6.

23. Orsini E, Giavaresi G, Trirè A, Ottani V, Salgar-ello S. Dental implant thread pitch and its influence on the osseointegration process: an in vivo comparison study. Int J Oral Maxillofac Implants. 2012;27:383-92.
24. Lan T, Du J, Pan C, Lee H, Chung W. Biomechanical analysis of alveolar bone stress around implants with different thread designs and pitches in the mandibular molar area. Clin Oral Investig. 2012;16:363-9.

25. Kong L, Liu BL, Hu KJ, Li DH. Song YL. Ma P, et al. Optimized thrend pitch design and stress analysis of the cylinder screwed dental implant. Hua Xi Kou Qiang Yi Xue Za Zhi. 2006:24:509-12.

26. Abuhussein H, Pagni G, Rebaudi A, Wang H. The effect of thread pattern upon implant osseointegration. Clin Oral Implants Res. 2010;21:129-36.

27. Gehrke SA, Marin GW. Biomechanical evaluation of dental implants with three different designs: Removal torque and resonance frequency analysis in rabbits. Ann Anat. 2015;199:30-5.

28. Sabeva E, Peev S, Miteva M, Georgieva M. The impact of the thread design compared to the impact of the surface topography on the primary stability of implants inserted into fresh pig ribs. Scr Sci Med Dent. 2017;3:60-4.

29. McCullough J, Klokkevold P. The effect of implant macro-thread design on implant stability in the early postoperative period: a randomized, controlled pilot study. Clin Oral Implants Res. 2017;28:1218-26.

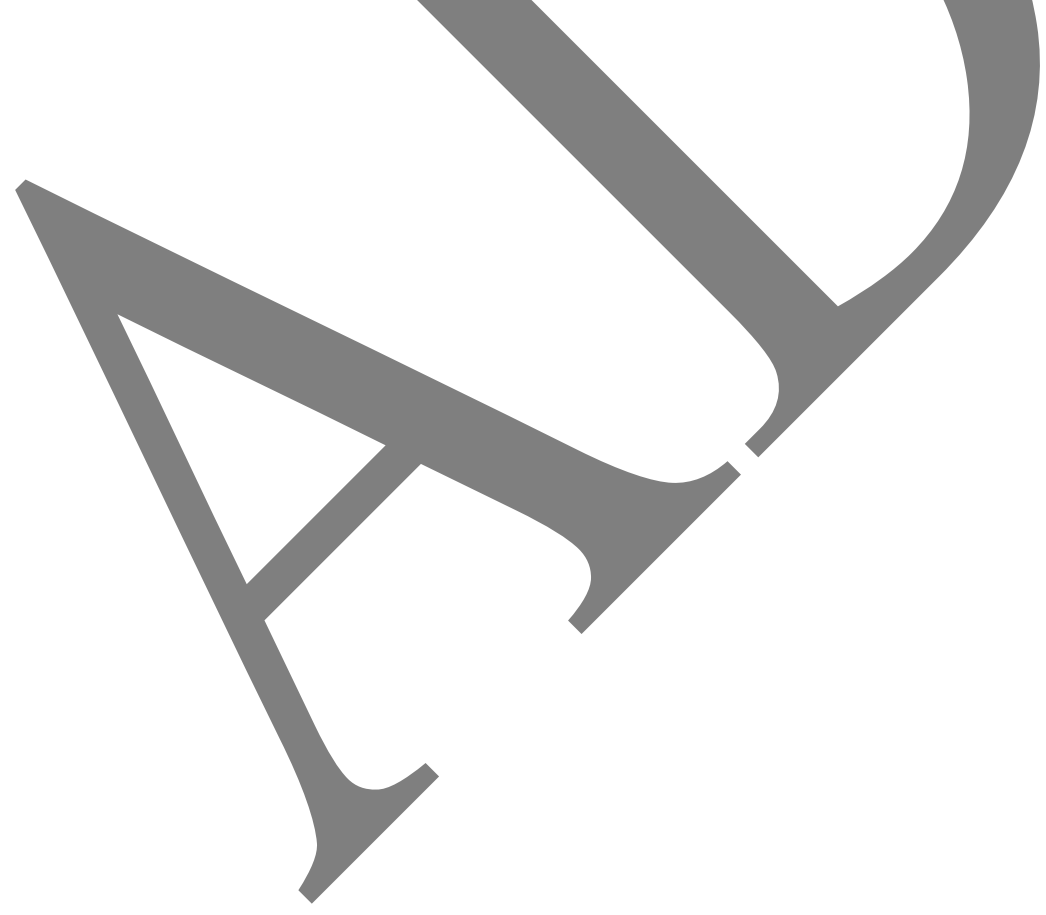

